\title{
Electron-phonon energy transfer in hot-carrier solar cells
}

\author{
Antonio Luque, Antonio Martí * \\ Instituto de Energía Solar, Universidad Politécnica de Madrid, Escuela Técnica Superior de Ingenieros de Telecomunicación Ciudad Universitaria sn, 28040 Madrid, Spain
}

A R T I C L E I N F O

Keywords:

Hot-carriers solar cells

Novel concepts

Electron-phonon interaction

\begin{abstract}
A B S T R A C T
Hot-carrier solar cells may yield very high efficiency if the heat transfer from electrons to phonons is low enough. In this paper we calculate this heat transfer for the two inelastic mechanisms known to limit the electric conductivity: the multi-valley scattering in non-polar semiconductors and the coupling of electrons to longitudinal optical phonons in polar semiconductors. Heat transfer is ruled by matrix elements deduced from electric conductivity measurements. The cell power extracted from hotcarrier solar cells affected by this mechanism, but otherwise ideal, is deduced. It is found that Si and Ge solar cells, mainly under concentrated sun light, might lead to better efficiencies than conventional cells.
\end{abstract}

\section{Introduction}

A detailed balance limit for the conversion efficiency of solar cells was established in 1961 by Shockley and Queisser [1] and reviewed in Ref. [2]. This model is based on considering radiative recombination as the ultimate unavoidable losses mechanism of the cell. For the case of maximum concentration (the one producing isotropic illumination with the sun's photosphere brightness) this limit is $40.7 \%$ for $6000 / 300 \mathrm{~K}$ Sun/Earth temperatures. This is well below the efficiency limit of a reversible solar device [3] (93.3\%) and the main reason is because, in a solar cell, photons below the band gap are not absorbed and, of those of higher energy, at most, only the band gap energy (times the Carnot Factor) can be extracted because carriers are supposed to become quickly thermalised within their energy band.

The concept of hot-carrier (HC) solar cell was proposed to overcome this situation [4,5]. In the $\mathrm{HC}$ solar cell, the electrons are thermally isolated from the phonons and they may stay at a different temperature. Furthermore, very narrow energy-selective contacts are used that contribute to the thermal insulation of the electrons. In this way, the ideal $\mathrm{HC}$ solar cell has a detailed balance efficiency limit [3] of $85.4 \%$.

But electrons in solids are soaked in a fluid of phonons and, at a first glance, isolating the electrons from the phonons may appear very difficult.

Slow phonon thermalisation [6] has been observed in some nano-structured materials and it might perhaps appear in some large band gap bulk materials like InN with large phononic band

\footnotetext{
* Corresponding author. Tel.: + 34914533551 ; fax: +34915446341.

E-mail address: amarti@etsit.upm.es (A. Martí).
}

gap [7,8]. No HC solar cell has been fabricated so far but an elegant concept has been suggested $[9,10]$ for the thermal isolation of the electrons consisting of avoiding the thermal contact between acoustic and optical phonons while the latter are kept at the electron temperature. We think that the confirmation of the feasibility of this mechanisms will require to determine the extent to which the an-harmonic interaction [11,12], among others, would allow the existence of two different temperatures for the phonons. In addition, the group velocity of the optical phonons at high temperatures should be small enough as to make negligible the thermal conductivity due to these phonons. Indeed, the concept deserves further research.

However other devices, like the Gunn [13] or the Read [14] diodes, to give some few examples, are based on hot or warm electrons that appear at high electrical currents in GaAs or at the avalanche breakdown in reverse biased $\mathrm{p}-\mathrm{n}$ diodes. Therefore, a more straightforward analysis of the possibility of isolating hot electrons from phonons is opportune.

It would be illusory to pretend preventing any interaction between electrons and phonons in a solid. The density of phonon modes is three times the density of atoms in the semiconductor; at room temperature every mode contains, in average, several phonons. This density is several orders of magnitude above the density of electrons in a non-degenerate semiconductor. In consequence, frequent collisions are produced between electrons and phonons. Many of these collisions, however, are deemed to be elastic [15] and they are not to be avoided in the HC solar cell. Only the inelastic collisions that occur mainly with optical phonons or high-energy acoustic ones, are to be avoided. The purpose of this paper is to examine to which extent the electrons in a solar cell can be kept hot and how the heat transfer from the electrons to the phonons through inelastic scattering mechanisms 
affect the behaviour of the otherwise ideal HC solar cell. Obviously, once the mechanisms are identified, it is not less important to suggest the appropriate materials.

Much of our knowledge concerning elastic and non-elastic electron-phonon interaction comes from the study of the electric conductivity and the Hall effect. In these researches it was very early recognised that a basic mechanism limiting the mobility at low temperatures was the elastic scattering by charged impurities [16]. In contrast, at room and higher temperatures, the dominating factor was first attributed to non-elastic scattering by acoustic phonons associated with the band gap changes (deformation potential) produced by the compression and expansion of the lattice under the effect of long wavelength vibrations of the lattice (low-energy acoustic phonons) [17,18]. However, this mechanism does not explain the temperature variation of the electric mobility in most materials.

Most elemental semiconductors, such as $\mathrm{Si}$ and $\mathrm{Ge}$, are of indirect band gap and, in them, multi-valley scattering [19] with phonons of large q-vector, that is a non-elastic mechanism, plays an important role and is taken into account to fit the calculated and the observed mobilities at room and higher temperatures. (e.g. see Fig. 4 in Ref. [20]).

In polar semiconductors, the dominating mechanism was found to be associated to the strong interaction [21] of the electrons with longitudinal optical phonons producing electric dipoles in their vibration (see Fig. 3 in Ref. [22]). This mechanism is inelastic as it involves the generation and annihilation of optical phonons of high energy.

Other scattering mechanisms have been identified (see for instance Ref. [15], Section 32.1) but they are not dominant in the mobility studies and we shall not consider them in this paper.

The matrix elements of the interactions described, obtained in part from fitting to mobility experiments, will be used in this paper to calculate the power interchange between electrons and phonons when they are at different temperatures. Then, the heat loss of the electrons based on these mechanisms will be introduced in the otherwise ideal power expression of the $\mathrm{HC}$ solar cell and the performance limitations will be discussed.

\section{Thermal coupling}

Under the effect of a force on the electrons, e.g. an electric field, the Fermi Dirac (FD) probability of occupation of the electrons becomes distorted. This distortion is balanced by a tendency to return to the equilibrium due to elastic and inelastic collisions of a variety of natures. For each type of collisions, the following equation can be written

$\left.\frac{\delta f(\mathbf{k})}{\delta t}\right|_{\text {coll }}=-\frac{\Omega}{8 \pi^{3}} \int\left[S\left(\mathbf{k}, \mathbf{k}^{\prime}\right) f(\mathbf{k})\left(1-f\left(\mathbf{k}^{\prime}\right)\right)-S\left(\mathbf{k}^{\prime}, \mathbf{k}\right) f\left(\mathbf{k}^{\prime}\right)(1-f(\mathbf{k}))\right] d^{3} k^{\prime}$

where $\Omega$ is the volume of the crystal, $S\left(\mathbf{k}, \mathbf{k}^{\prime}\right)$ the scattering probability for passing from state $\mathbf{k}$ to state $\mathbf{k}^{\prime}$ and $f(\mathbf{k})$ the distorted probability of occupation of a single electron state in a band, characterised by a $\mathbf{k}$-vector and a spin. If the spin has no effect in the scattering then the state can be characterised by the vector $\mathbf{k}$ alone and a factor 2 will precede the integral.

The scattering probability is determined by the Fermi Golden Rule as

$S\left(\mathbf{k}, \mathbf{k}^{\prime}\right)=\frac{2 \pi}{\hbar}\left|M\left(\mathbf{k}, \mathbf{k}^{\prime}\right)\right|^{2} \delta\left(E_{\mathbf{k}}-E_{\mathbf{k}^{\prime}} \pm \Delta E_{\mathbf{k}, \mathbf{k}^{\prime}}\right)$

where $M\left(\mathbf{k}, \mathbf{k}^{\prime}\right)$ is the element of matrix of the Hamiltonian perturbation describing the scattering and $\Delta E_{\mathbf{k}, \mathbf{k}^{\prime}}$ is the change of electronic energy involved in the transition.
Under an electric field, the distorted occupation probability becomes anisotropic. This leads to significant complexities. To obtain the Boltzmann equation a linearization process is used leading to the introduction of a collision time. In our case, the distorted probability of occupation will be described by a temperature $T_{h c}$ different from the phonon temperature $T_{a}$, no anisotropy is produced and the treatment is much easier.

We are interested in the transference of heat from the electrons to the phonons or vice-versa. We shall use the simplifying assumption, also common in the mobility studies, that the energy of the phonons under consideration is the same for all the electronic transitions.

This transference of energy is actually ruled by the following equation:

$$
\begin{aligned}
W(\mathbf{k}) /\left(\frac{2 \pi}{\hbar} \frac{2}{8 \pi^{3}}\right) \\
=\int\left[\hbar \omega\left|M_{e}\left(\mathbf{k}, \mathbf{k}^{\prime}\right)\right|^{2} n f(\mathbf{k})\left(1-f\left(\mathbf{k}^{\prime}\right)\right)-\hbar \omega\left|M_{e}\left(\mathbf{k}^{\prime}, \mathbf{k}\right)\right|^{2}(n+1) f\left(\mathbf{k}^{\prime}\right)\right. \\
\quad \times(1-f(\mathbf{k}))] \delta\left(E_{\mathbf{k}}+\hbar \omega-E_{\mathbf{k}^{\prime}}\right) d^{3} \mathbf{k}^{\prime}-\int\left[\hbar \omega\left|M_{e}\left(\mathbf{k}, \mathbf{k}^{\prime}\right)\right|^{2}(n+1)\right. \\
\left.\quad \times f(\mathbf{k})\left(1-f\left(\mathbf{k}^{\prime}\right)\right)-\hbar \omega\left|M_{e}\left(\mathbf{k}^{\prime}, \mathbf{k}\right)\right|^{2} n f\left(\mathbf{k}^{\prime}\right)(1-f(\mathbf{k}))\right] \\
\quad \times \delta\left(E_{\mathbf{k}}-\hbar \omega-E_{\mathbf{k}^{\prime}}\right) d^{3} \mathbf{k}^{\prime}
\end{aligned}
$$

where, to the electron transition with absorption of one phonon and its reverse with emission, we add the electron transition with emission of one phonon (occurring only if the electron energy is above $h(\omega)$ and its reverse with absorption.

In the preceding expression $f(\mathbf{k})=f\left(E_{\mathbf{k}}\right)$ is the FD function at temperature $T_{h c}$ while the average number of phonons $n$ is given by the Bose Einstein (BE) function at ambient temperature $T_{a}$

Note that here we have separated the matrix element into two factors, one $\left|M_{e}\left(\mathbf{k}, \mathbf{k}^{\prime}\right)\right|=\left|M_{e}\left(\mathbf{k}^{\prime}, \mathbf{k}\right)\right|$, corresponding to the electronic part of the state and one $\sqrt{n}$ or $\sqrt{n+1}$, with $n$ the number of phonons in the mode, corresponding to the annihilation or creation of a phonon.

In the early studies, for the elastic collisions with acoustic phonons under the deformation potential, no phonon is absorbed or emitted in the transition and no energy transfer is produced in any scattering event. So the right hand term of the preceding calculation is identically zero. Only the inelastic transitions mentioned in the introduction will be considered here. Nevertheless, we shall bring here the matrix element of this transition, as presented by Bardeen and Shockley [17], because other matrix elements are, in some cases, referred to this one. This element is

$\left|M_{a d e f}\left(\mathbf{k}, \mathbf{k}^{\prime}\right)\right|^{2}=\frac{\Xi^{2} k T_{a}}{\Omega c_{l}}$

where $\Xi$ is the change in band gap per unit of strain, that is, $\Xi=\Omega d E_{G} / d \Omega$ and $c_{l}$ is the elastic constant for longitudinal deformation. It is closely related to the elastic components $c_{i k}$ of the elastic tensor (see Ref. [15], Section 32.2.1.C and Chapter 4). In high symmetry crystals this constant is related to the sound speed for longitudinal waves $v_{s l}=v_{s}=\sqrt{c_{l} / \rho}, \rho$ being the density. Sometimes $c_{l}$ in Eq. (4) is written (and data are given) in terms of $\rho$ and $v_{s}$. Note that this element of matrix is constant, independently of $\mathbf{k}$, $\mathbf{k}^{\prime}$.

\subsection{Scattering in multi-valley semiconductors}

Indirect gap semiconductors have multiple minima at the bottom of the conduction band. For instance, silicon has six minima in the $X$ points of the reciprocal lattice at about $0.8 \times(2 \pi / a) \times(1,0,0)$ and germanium has eight minima at the 
positions $(2 \pi / \mathrm{a}) \times(1 / 2,1 / 2,1 / 2)$, although these valleys are only half located inside the 1 st Brillouin zone, so giving an equivalent of four full valleys.

The electron here has a $\mathbf{k}$-vector not very different to the central one of the valley $\mathbf{K}_{i}$ in which the electron is located. By absorption of a phonon with $\mathbf{q}$ close to $\mathbf{K}_{j}-\mathbf{K}_{i}$ the electron acquires a $\mathbf{k}$-vector close to $\mathbf{K}_{j}$. For emission, the phonon must have $\mathbf{q} \cong \mathbf{K}_{j}-\mathbf{K}_{i}$. Since the $\mathbf{q}$ of the phonons involved in the transitions is closely the same for all of them, their energy $\hbar \omega(\mathbf{q})$ can be considered constant in all the process. An inter-valley deformation potential $D_{i}$ constant is defined that allows to fit the experimental data of the mobility and obtain the matrix element corresponding only to the electronic part of the Hamiltonian that becomes (see Eq. 32.57 in Ref. [15])

$\left|M_{\text {interv }}\left(\mathbf{k}, \mathbf{k}^{\prime}\right)\right|^{2}=\frac{D_{i}^{2} k T_{a}}{2 \Omega \rho \omega^{2}}$

where $\rho$ is the material density. Notice that also in this case the matrix element is independent of $\mathbf{k}$, $\mathbf{k}^{\prime}$. The integrand in Eq. (3) depends only on the energy and the integral in $d k^{\prime 3} / 4 \pi^{3}$ can be changed into an integral on $g\left(E_{\mathbf{k}^{\prime}}\right) d E_{\mathbf{k}^{\prime}}=g\left(E^{\prime}\right) d E^{\prime}$ by making use of the density of states

$g\left(E^{\prime}\right)=\frac{2^{1 / 2} \Omega m_{e}^{3 / 2}}{\pi^{2} \hbar^{3}} E^{1 / 2}$

where $m_{e}$ is the effective mass for density of states. This leads to

$$
\begin{aligned}
W(\mathbf{k})= & \frac{2^{1 / 2} D_{i}^{2} k T_{p h} m_{e}^{3 / 2}}{\pi \hbar^{4} \rho \omega^{2}}(E+\hbar \omega)^{1 / 2}[\hbar \omega n f(E)(1-f(E+\hbar \omega)) \\
& -\hbar \omega(n+1) f(E+\hbar \omega)(1-f(E))]-\frac{2^{1 / 2} D_{i}^{2} k T_{p h} m_{e}^{3 / 2}}{\pi \hbar^{4} \rho \omega^{2}} \\
& \times(E-\hbar \omega)^{1 / 2}[\hbar \omega(n+1) f(E)(1-f(E-\hbar \omega)) \\
& -\hbar \omega n f(E-\hbar \omega)(1-f(E))]
\end{aligned}
$$

We want to remind again that the $f$ are FD functions for temperature $T_{h c}$ and $n=n(h \omega)$ is the BE function for temperature $T_{a}$.

We assume that there are $Z$ minima in the conduction band. Although the phonon $\mathbf{q}$ associated with transitions between these minima can be somewhat different and thus $\hbar \omega(\mathbf{q})$ can also be somewhat different, we can neglect these differences and state that $W(\mathbf{k})$ is $Z-1$ times the one appearing in Eq. (7).

Integrating over all the $\mathbf{k}$ and taking into account that $W(\mathbf{k})=W\left(E_{\mathbf{k}}\right)$, so that we can use once again the function $g(E)$, and adding the results corresponding to the integration in each minimum (under the simplification of a common value of $h \omega$ ) we obtain

$\dot{Q}=Z(Z-1) \frac{2 m_{e}^{3} \Omega D_{i}^{2}\left(k T_{a}\right)\left(k T_{h c}\right)^{2}}{\pi^{3} \hbar^{5} \rho(\hbar \omega)}\left[n J_{o u t}^{a b s}-(n+1) J_{\text {in }}^{e m}-(n+1) J_{o u t}^{e m}+n J_{i n}^{a b s}\right]$

being

$$
\begin{aligned}
J_{\text {outt }}^{a b s}\left(\hbar \omega / k T_{h c}, E_{F} / k T_{h c}\right) & \int_{0}^{\infty} f\left(E / k T_{h c}\right)\left[1-f\left((E+\hbar \omega) / k T_{h c}\right)\right] \\
& \times \sqrt{\frac{E(E+\hbar \omega)}{\left(k T_{h c}\right)^{2}} \frac{d E}{k T_{h c}} j_{i n}^{e m}\left(\hbar \omega / k T_{h c}, E_{F} / k T_{h c}\right)} \\
= & \int_{0}^{\infty} f\left((E+\hbar \omega) / k T_{h c}\right)\left(1-f\left(E / k T_{h c}\right)\right) \\
& \times \sqrt{\frac{E(E+\hbar \omega)}{\left(k T_{h c}\right)^{2}}} \frac{d E}{k T_{h c}} J_{o u t}^{e m}\left(\hbar \omega / k T_{h c}, E_{F} / k T_{h c}\right)
\end{aligned}
$$

$$
\begin{aligned}
= & \int_{\hbar \omega / k T_{h c}}^{\infty} f\left(E / k T_{h c}\right)\left[1-f\left((E-\hbar \omega) / k T_{h c}\right)\right] \\
& \times \sqrt{\frac{E(E-\hbar \omega)}{\left(k T_{h c}\right)^{2}}} \frac{d E}{k T_{h c}} J_{i n}^{a b s}\left(\hbar \omega / k T_{h c}, E_{F} / k T_{h c}\right) \\
= & \int_{\hbar \omega / k T_{h c}}^{\infty} f\left((E-\hbar \omega) / k T_{h c}\right)\left(1-f\left(E / k T_{h c}\right)\right) \\
& \times \sqrt{\frac{E(E-\hbar \omega)}{\left(k T_{h c}\right)^{2}}} \frac{d E}{k T_{h c}}
\end{aligned}
$$

In Eq (8), it results clear that the absorption of phonons heats the electrons while the emission of phonons cools them. Although it does not appear explicitly, the integrals in Eq. (9) are function of the Fermi level through the function $f$.

We present in Fig. 1 the power collected by the electrons (delivered when negative) corresponding to $\mathrm{Ge}, \mathrm{Si}$ and $\mathrm{GaP}$ with a volume of $1 \mathrm{~cm}^{2} \times 1 \mu \mathrm{m}$. Parameters in Table 1 have been used [20] in the calculations. Ge and Si have been selected because they are semiconductors in which multi-valley scattering affects the mobility at high temperatures. The thickness used is probably insufficient for indirect gap semiconductors to achieve full absorption but ideally it is not so far: ergodic light confinement [23] can produce an equivalent thickness of $4 n_{r}^{2}$, about 50-60 times the physical size.

The results are very critically dependent on the density of electrons in the conduction band that is introduced in the formulas indirectly through the Fermi level. The power loss is proportional to the density of electrons. To reduce the power loss this density must be very small. In the calculations we have considered the Fermi level as determined by an ionized acceptor density of $5 \times 10^{18} \mathrm{~cm}^{-3}$ and taking into account also the electron temperature.

The lowest loss of power is for GaP because of its low number of minority electrons due to the larger band gap. However, this semiconductor is not very adequate because it is transparent to a large part of the solar spectrum and, furthermore, it is polar leading to additional non-elastic mechanisms not calculated here.

We define the net phonon emission time, $\tau_{\text {coll }}$, by the following equation ( $n_{e l}$ is the density of electrons):

$\dot{Q}=\frac{\hbar \omega n_{e l} \Omega}{\tau_{c o l l}}$

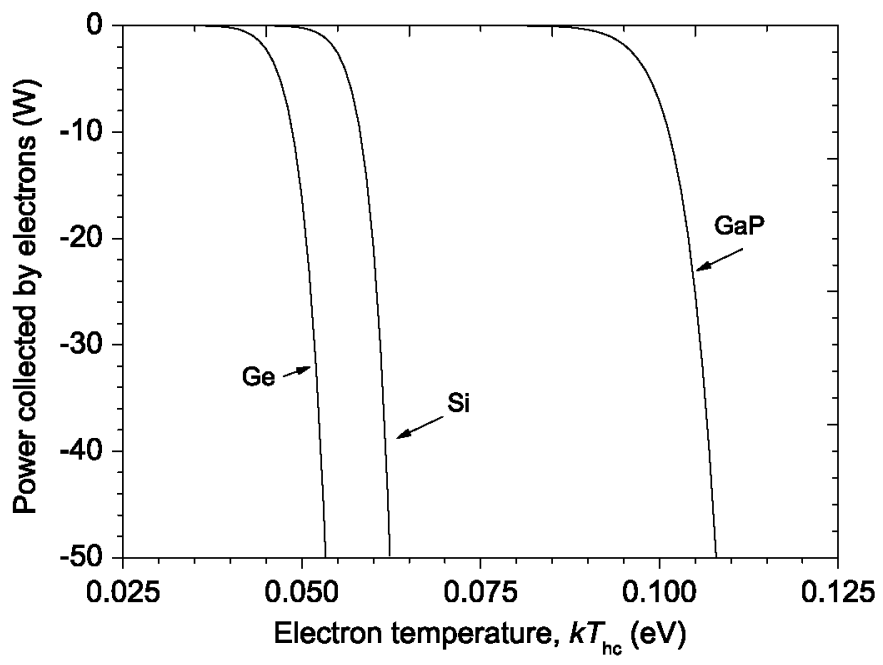

Fig. 1. Power collected by the electrons (negative for $T_{h c}>T_{a}$ ) for a phonon temperature $T_{a}=300 \mathrm{~K}$. Parameters in Table 1 have been used for the calculations. 


\begin{tabular}{|c|c|c|c|}
\hline & \multicolumn{3}{|l|}{ Material } \\
\hline & $\mathrm{Ge}$ & $\mathrm{Si}$ & GaP \\
\hline Conduction band minima, $Z$ & 4 & 6 & 3 \\
\hline Indirect band gap $E_{g}$ at $300 \mathrm{~K}(\mathrm{eV})$ & 0.664 & 1.12 & 2.25 \\
\hline Electron effective mass $\left(m_{e} / m_{0}\right)$ & 0.22 & 0.39 & 0.365 \\
\hline Hole effective mass $\left(m_{h} / m_{0}\right)$ & 0.18 & 0.591 & 0.514 \\
\hline Material density $\left(\mathrm{kg} \mathrm{m}^{-3}\right)$ & 5320 & 2330 & 4130 \\
\hline Inter-valley phonon energy (e.V.) & 0.0329 & 0.0467 & 0.0309 \\
\hline Acoustic phonon deformation potential, $\Xi(\mathrm{eV})$ & 9 & 6.5 & 13 \\
\hline Inter-valley phonon deformation potential, $D_{i}\left(\mathrm{eV} \mathrm{m}^{-1}\right)$ & $3 \times 10^{10}$ & $3 \times 10^{10}$ & $1.2 \times 10^{11}$ \\
\hline Longitudinal deformation $\left(\begin{array}{lll}1 & 1 & 1\end{array}\right)$ direction $\left(\mathrm{Nm}^{-2}\right)$ & $1.64 \times 10^{11}$ & $2.1 \times 10^{11}$ & $1.83 \times 10^{11}$ \\
\hline Density of acceptors $\left(\mathrm{cm}^{-3}\right)$ & $5 \times 10^{18}$ & $5 \times 10^{18}$ & $5 \times 10^{18}$ \\
\hline Volume of the sample $\Omega\left(\mathrm{m}^{3}\right)$ & $10^{-10}$ & $10^{-10}$ & $10^{-10}$ \\
\hline
\end{tabular}

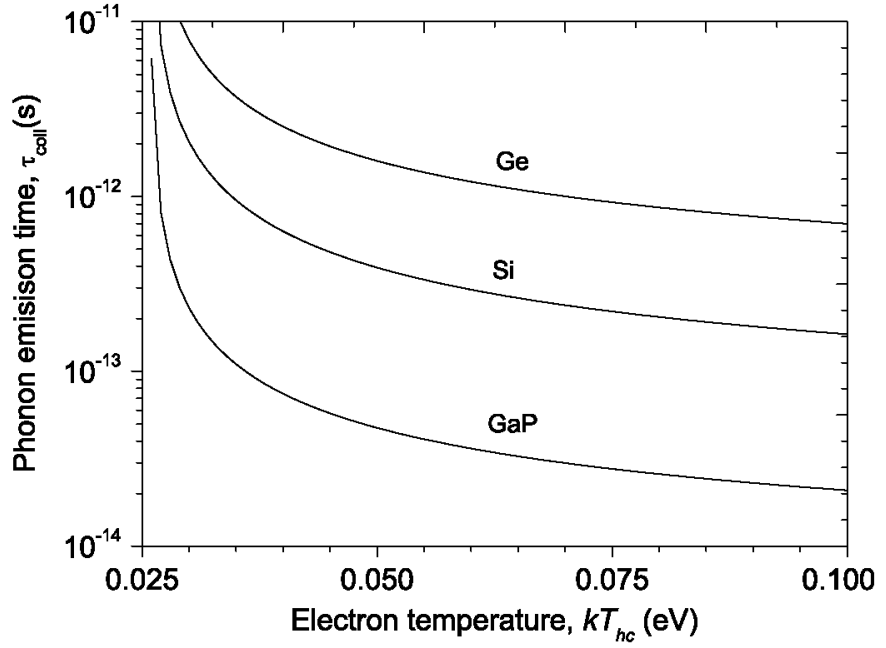

Fig. 2. Phonon emission time vs. electron temperature for a phonon temperature $T_{a}=300 \mathrm{~K}$ calculated with parameters in Table 1 .

The word net refers to the fact that absorptions and emissions are considered although the second mechanism dominates. A plot of it is given in Fig. 2. It can be seen that, for Ge, it is of several picoseconds and, for $\mathrm{Si}$ and $\mathrm{GaP}$, it is below the picosecond for high enough electron temperature. This time can be compared in Table 2 to the collision time (elastic and inelastic) determining the mobility $\left(\mu=e \tau / m_{e}\right)$. Conceptually, the two times are different because the unbalance of the distribution function is different; furthermore the phonon emission mean time depends on the electron temperature. Nevertheless, for Si and GaP and for the temperature chosen, they are rather similar.

In the case of $\mathrm{Si}$, with a dependence $T^{-2.5}$, it is known that the mobility is rather affected by multi-valley scattering. In the case of $\mathrm{Ge}$, the dependence is $T^{-1.63}$, very close to the $T^{-3 / 2}$ law that characterises the elastic collisions with acoustic phonons. This means that this mechanism dominates as suggested by the value of the phonon emission time, which in this case is much longer.

Also in Table 2 we can see that the multi-valley matrix element is well correlated with the phonon emission time (the matrix element decreases when the phonon emission time increases). It is not the only factor intervening but it is an important one. The correlation is very strong with the ratio between multi-valley and acoustic matrix elements. This ratio is strongly correlated with the mobility temperature dependence (actually the matrix element parameters are fitted with it) and it is also well correlated with our calculated phonon emission time.

\subsection{Scattering with optical phonons in polar semiconductors}

In polar semiconductors, there is a strong coupling between the longitudinal optical phonons and the electrons. This means that the processes of energy interchange of energy between electrons and optical phonons is strong with emission and absorption of the later.

This interaction may happen at any $\mathbf{q - v e c t o r}\left(\mathbf{q}=\mathbf{k}^{\prime}-\mathbf{k}\right)$ but it is stronger with optical phonons of low $\mathbf{q}$-vector. The electronic part of the element of matrix is [22]

$\left|M_{\text {polar }}\left(\mathbf{k}, \mathbf{k}^{\prime}\right)\right|^{2}=\frac{e^{2} \hbar \omega}{4 \Omega \varepsilon_{0}}\left(\frac{1}{\varepsilon_{\text {opt }}}-\frac{1}{\varepsilon_{s t}}\right) \frac{1}{\left|\mathbf{k}-\mathbf{k}^{\prime}\right|^{2}}$

In this part of the paper we restrict our interest to direct band gap polar semiconductors, such as GaAs having the electrons at low $\mathbf{k}$ and therefore favouring the coupling.

For the calculation of Eq. (3) we shall consider spherical symmetry for the energy bands which is a reasonable approximation in direct gap semiconductors. We have to consider that now the matrix element is not independent of $\mathbf{k}$, $\mathbf{k}$. In spherical coordinates, Eq. (3) is written ( $\theta$ is the angle of $\mathbf{k}$ with $\mathbf{k}^{\prime}$ )

$$
\begin{aligned}
W(\mathbf{k})= & \Gamma \int \frac{\left[\hbar \omega n f\left(E_{\mathbf{k}}\right)\left(1-f\left(E_{\mathbf{k}^{\prime}}\right)\right)-\hbar \omega(n+1) f\left(E_{\mathbf{k}^{\prime}}\right)\left(1-f\left(E_{\mathbf{k}}\right)\right)\right]}{k^{2}+k^{2}-2 k k^{\prime} \cos \theta} \\
& \times \delta\left(E_{\mathbf{k}}+\hbar \omega-E_{\mathbf{k}^{\prime}}\right) k^{\prime 2} \sin \theta^{\prime} d \varphi^{\prime} d \theta^{\prime} d k^{\prime} \\
& -\Gamma \int \frac{\left[\hbar \omega(n+1) f\left(E_{\mathbf{k}}\right)\left(1-f\left(E_{\mathbf{k}^{\prime}}\right)\right)-\hbar \omega n f\left(E_{\mathbf{k}^{\prime}}\right)\left(1-f\left(E_{\mathbf{k}}\right)\right)\right]}{\mathbf{k}^{2}+\mathbf{k}^{2}-2 \mathbf{k k}^{\prime} \cos \theta} \\
& \delta\left(E_{\mathbf{k}}-\hbar \omega-E_{\mathbf{k}^{\prime}}\right) k^{\prime 2} \sin \theta^{\prime} d \varphi^{\prime} d \theta^{\prime} d k^{\prime} \\
& \text { with } \quad \Gamma=\frac{e^{2} \omega}{8 \pi^{2} \Omega \varepsilon_{0}}\left(\frac{1}{\varepsilon_{o p t}}-\frac{1}{\varepsilon_{s t}}\right)
\end{aligned}
$$

Assuming a spherical effective mass so that $E=\hbar^{2} k^{2} / 2 m_{e}$, $k=\sqrt{2 m_{e} E} / h$ and $d k / d E=\sqrt{m_{e}} / \hbar \sqrt{2 E}$ we obtain after some manipulation

$$
\begin{aligned}
W(\mathbf{k})= & \sqrt{2 m_{e}} \frac{\Gamma}{\hbar} \\
& \times \frac{\left[n \hbar \omega f\left(E_{\mathbf{k}}\right)\left(1-f\left(E_{\mathbf{k}}+\hbar \omega\right)\right)-(n+1) \hbar \omega f\left(E_{\mathbf{k}}+\hbar \omega\right)\left(1-f\left(E_{\mathbf{k}}\right)\right)\right]}{\sqrt{E_{k}}} \\
& \times \ln \left(\frac{\sqrt{E_{\mathbf{k}}+\hbar \omega}+\sqrt{E_{\mathbf{k}}}}{\left.\sqrt{E_{\mathbf{k}}+\hbar \omega}-\sqrt{E_{\mathbf{k}}}\right)-\sqrt{2 m_{e}} \frac{\Gamma}{\hbar}}\right. \\
& \times \frac{\left[(n+1) \hbar \omega f\left(E_{\mathbf{k}}\right)\left(1-f\left(E_{\mathbf{k}}-\hbar \omega\right)\right)-n \hbar \omega f\left(E_{\mathbf{k}}-\hbar \omega\right)\left(1-f\left(E_{\mathbf{k}}\right)\right)\right]}{\sqrt{E_{k}}} \\
& \times \ln \left(\frac{\sqrt{E_{\mathbf{k}}}+\sqrt{E_{\mathbf{k}}-\hbar \omega}}{\sqrt{E_{\mathbf{k}}}-\sqrt{\left(E_{\mathbf{k}}-\hbar \omega\right)}}\right)
\end{aligned}
$$




\begin{tabular}{|c|c|c|c|}
\hline & \multicolumn{3}{|l|}{ Material } \\
\hline & Ge & $\mathrm{Si}$ & GaP \\
\hline Electron mobilities at $300 \mathrm{~K}\left(\mathrm{~cm}^{2} \mathrm{~V}^{-1} \mathrm{~s}^{-1}\right)$ [20] & 3800 & 1750 & 200 \\
\hline Mobility collision time $\tau=\mu m_{e} / e(s)$ & $4.75 \times 10^{-13}$ & $3.88 \times 10^{-13}$ & $4.15 \times 10^{-14}$ \\
\hline Phonon emission time $\tau_{\text {coll }}$ at $k T_{e l}=0.05 \mathrm{e} . \mathrm{V}$. (s) & $1.60 \times 10^{-12}$ & $3.92 \times 10^{-13}$ & $4.75 \times 10^{-14}$ \\
\hline$\frac{2 \pi}{\hbar}\left|M_{\text {adef } f}\left(\mathbf{k}, \mathbf{k}^{\prime}\right)\right|^{2}=\frac{2 \pi}{\hbar} \frac{\Xi^{2} k T_{p h}}{\Omega c_{l}}\left(\mathrm{e} . \mathrm{V} . \mathrm{s}^{-1}\right)$ & $1.95 \times 10^{-4}$ & $7.95 \times 10^{-5}$ & $3.65 \times 10^{-4}$ \\
\hline$\frac{2 \pi}{\hbar}\left|M_{\text {interv }}\left(\mathbf{k}, \mathbf{k}^{\prime}\right)\right|^{2}=\frac{2 \pi}{\hbar} \frac{\hbar^{2} D_{i}^{2} k T_{p h}}{2 \Omega \rho(\hbar \omega)^{2}}\left(\right.$ e.V. $\left.s^{-1}\right)$ & $1.34 \times 10^{-5}$ & $1.52 \times 10^{-5}$ & $3.12 \times 10^{-4}$ \\
\hline$\left|M_{\text {interv }}\left(\mathbf{k}, \mathbf{k}^{\prime}\right)\right|^{2} /\left|M_{\text {adef } f}\left(\mathbf{k}, \mathbf{k}^{\prime}\right)\right|^{2}$ & 0.0685 & 0.1907 & 0.8566 \\
\hline
\end{tabular}

The next step is to integrate this along all the values of $\boldsymbol{k}$ :

$\dot{Q}=\frac{\Omega}{4 \pi^{3}} \int W(\mathbf{k}) d^{3} k=\frac{2^{1 / 2} m_{e}^{3 / 2} \Omega}{\hbar^{3} \pi^{2}} \int W\left(E_{\mathbf{k}}\right) \sqrt{E_{\mathbf{k}}} d E$

We can separate the heat transfer rate into for components: $I_{\text {out }}^{a b s}, I_{\text {in }}^{e m}, I_{\text {out }}^{e m}, I_{\text {in }}^{a b s}$

$$
\begin{aligned}
I_{\text {out }}^{a b s} & =\int_{0}^{\infty} f\left(E_{\mathbf{k}}\right)\left(1-f\left(E_{\mathbf{k}}+\hbar \omega\right)\right) \ln \left(\frac{\sqrt{E_{\mathbf{k}}+\hbar \omega}+\sqrt{E_{\mathbf{k}}}}{\sqrt{E_{\mathbf{k}}+\hbar \omega}-\sqrt{E_{\mathbf{k}}}}\right) \frac{d E}{k T_{h c}} I_{1 n}^{e m} \\
& =\int_{0}^{\infty} f\left(E_{\mathbf{k}}+\hbar \omega\right)\left(1-f\left(E_{\mathbf{k}}\right)\right) \ln \left(\frac{\sqrt{E_{\mathbf{k}}+\hbar \omega}+\sqrt{E_{\mathbf{k}}}}{\sqrt{E_{\mathbf{k}}+\hbar \omega}-\sqrt{E_{\mathbf{k}}}}\right) \frac{d E}{k T_{h c}} I_{o u t}^{e m} \\
& =\int_{\hbar \omega / k T_{h c}} f\left(E_{\mathbf{k}}\right)\left(1-f\left(E_{\mathbf{k}}-\hbar \omega\right)\right) \ln \left(\frac{\sqrt{E_{\mathbf{k}}}+\sqrt{E_{\mathbf{k}}-\hbar \omega}}{\sqrt{E_{\mathbf{k}}}-\sqrt{E_{\mathbf{k}}-\hbar \omega}}\right) \frac{d E}{k T_{h c}} I_{\text {in }}^{a b s} \\
& =\int_{\hbar \omega / k T_{h c}}^{\infty} f\left(E_{\mathbf{k}}-\hbar \omega\right)\left(1-f\left(E_{\mathbf{k}}\right)\right) \ln \left(\frac{\sqrt{E_{\mathbf{k}}}+\sqrt{E_{\mathbf{k}}-\hbar \omega}}{\sqrt{E_{\mathbf{k}}}-\sqrt{E_{\mathbf{k}}-\hbar \omega}}\right) \frac{d E}{k T_{h c}}
\end{aligned}
$$

So that

$\dot{Q}=\frac{m_{e}^{2} e^{2}(\hbar \omega)^{2} \Omega\left(k T_{h c}\right)}{4 \hbar^{5} \pi^{4} \varepsilon_{0}}\left(\frac{1}{\varepsilon_{o p t}}-\frac{1}{\varepsilon_{s t}}\right)\left[n I_{o u t}^{a b s}-(n+1) I_{\text {in }}^{e m}-(n+1) I_{\text {out }}^{e m}+n I_{i n}^{a b s}\right]$

Note, by a simple change of variables $(E \rightarrow E+h \omega)$ that the integrals $I_{\text {out }}^{a b s}=I_{\text {in }}^{a b s}=I_{a b s}$ and the same for $I_{\text {in }}^{e m}=I_{\text {out }}^{e m}=I_{\text {em }}$.

A similar treatment can be done for the holes in the valence band. Actually we find in it two separated bands that might not be degenerated at the point $\Gamma$. We can treat each band separately and then add the power losses.

We present in Fig. 3 the power collected by the electrons (lost when negative) for several polar semiconductors considering both the conduction and valence bands. Again this loss is very sensible to the density of carriers but now we cannot reduce this density by setting the Fermi level near the valence band. The calculations presented correspond to the Fermi level position of the intrinsic semiconductor and is variable with the temperature. The parameters used are those in Table 3 .

According to this picture, the most favourable material is the GaAs followed by CdTe but this is again because the band gap is larger and the density of carriers is smaller. The behaviour of the InAs is very poor. It has been included to see the effect of a small band gap semiconductor.

In the preceding calculations, the semiconductor has been considered intrinsic. For an electron temperature of $0.05 \mathrm{eV}$ the power delivered by the electrons (the opposed to the power

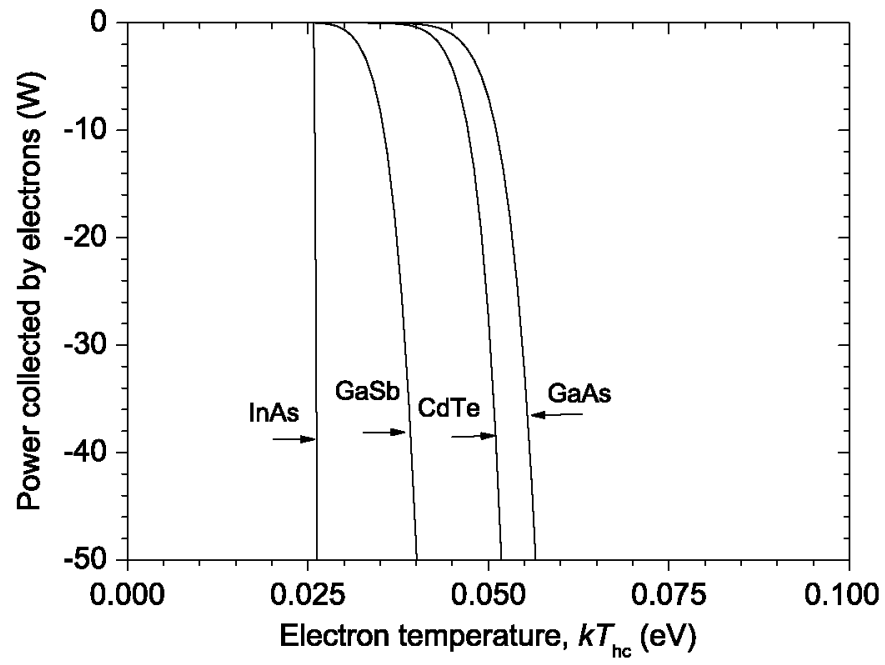

Fig. 3. Power collected by the electrons taking into account phonon scattering with the conduction and two valence bands electrons (negative for $T_{h c}>T_{a}$ ) calculated for a phonon temperature $T_{a}=300 \mathrm{~K}$ and using parameters in Table 3 . Semiconductors are considered intrinsic.

collected, negative) has been calculated in Fig. 4 vs. the position of the Fermi level. When the Fermi level approaches to the valence band (right in the graphic) they are the holes loosing more energy while, when it approaches to the conduction band, the energy is lost by the conduction band electrons. Since there are fewer states in the conduction band, the optimum is a bit displaced towards the conduction band, as well as the optimum position of the intrinsic Fermi level. The optimum position of the Fermi level is not exactly the one of the intrinsic Fermi level but it is very close to it. Note that the electron temperature is taken different for each material, approximately where the maximum power point of the cells is located in the analysis to follow.

The net phonon emission time, defined by Eq. (10), is represented in Fig. 5 now limited to phonon emissions by electrons in the conduction band. For not very polar semiconductors (III-V) they are above most of the values in Fig. 2 corresponding to the multi-valley scattering, and are comparable to Ge. This is indeed associated to the high mobility of all these materials. We can observe that the longest lifetime corresponds to GaSb mostly due to its low polarity and reflected by the low difference between static and optic dielectric constants. Nevertheless, the power delivered by the electrons is high due to its high electron density, consequence of its narrow 


\begin{tabular}{|c|c|c|c|c|}
\hline & \multicolumn{4}{|c|}{ Materials } \\
\hline & GaAs & InAs & GaSb & CdTe \\
\hline Direct band gap $E_{g}$ at $300 \mathrm{~K}(\mathrm{eV})$ (Ref. [15] Table 9.8) & 1.411 & 0.356 & 0.70 & 1.43 \\
\hline Electron effective mass $\left(m_{e} / m_{0}\right)$ (Ref. [15] Table 9.3) & 0.067 & 0.024 & 0.041 & 0.096 \\
\hline \multicolumn{5}{|l|}{ Hole effective masses (Table 9.6 in Ref. [15] and Eq. 9.12): } \\
\hline$\left(m_{l h} / m_{0}\right)$ & 0.0831 & 0.0265 & 0.0714 & 0.1111 \\
\hline$\left(m_{h h} / m_{0}\right)$ & 0.538 & 0.333 & 0.0794 & 0.625 \\
\hline $\begin{array}{l}\text { Hole mass for DOS } \\
\left(m_{i h}^{3 / 2}+m_{h h}^{3 / 2}\right)^{2 / 3} / m_{0}\end{array}$ & 0.559 & 0.338 & 0.120 & 0.656 \\
\hline $\begin{array}{l}\text { Hole mass for energy transfer } \\
\left(m_{l h}^{2}+m_{h h}^{2}\right)^{1 / 2} / m_{0}\end{array}$ & 0.544 & 0.334 & 0.107 & 0.635 \\
\hline Static dielectric constant $\varepsilon_{s t}$ (Ref. [15] Table 14.1) & 13.1 & 14.61 & 15.69 & 10.3 \\
\hline Optic dielectric constant $\varepsilon_{\text {opt }}$ (Ref. [15] Table 14.1) & 11.1 & 11.8 & 14.44 & 6.9 \\
\hline$\left(1 / \varepsilon_{o p t}-1 / \varepsilon_{s t}\right)^{-1}$ & 118.9 & 61.4 & 151.6 & 20.9 \\
\hline Optical phonon energy (e.V.) (Ref. [15] Table 5.1) & 0.0352 & 0.0295 & 0.0287 & 0.210 \\
\hline Volume of the sample $\Omega\left(\mathrm{m}^{3}\right)$ & $10^{-10}$ & $10^{-10}$ & $10^{-10}$ & $10^{-10}$ \\
\hline
\end{tabular}

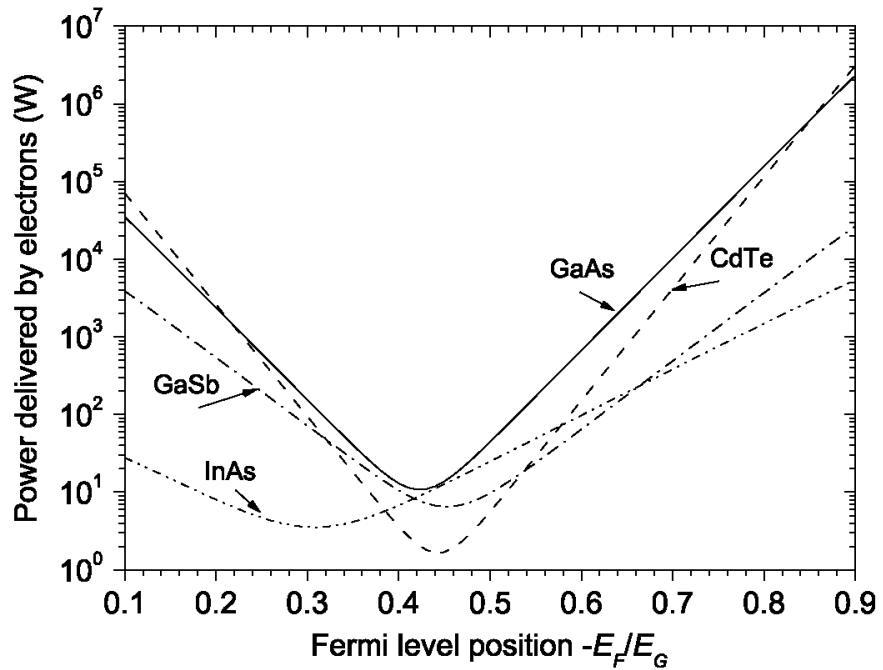

Fig. 4. Power lost (not collected) by the electrons for a phonon temperature $T_{a}=300 \mathrm{~K}$, electron temperature $k T_{h c}=0.05 \mathrm{eV}$ and calculated for parameters in Table 3 as a function of the position of the Fermi level $E_{F}$ along the band gap. The electron temperature is taken (arbitrarily) as 600 (for GaAs), 300 (for InAs), 400 (for GaSb), $500 \mathrm{~K}$ (for CdTe) and the intrinsic level $E_{F} / E_{G}$ is located at -0.442 , $-0.356,-0.460,-0.457 \mathrm{eV}$, respectively.

band gap. CdTe results the one with smallest phonon emission time as a consequence of its high polarity, characteristic of II-VI compounds and reflected in the large difference in dielectric constants. Similar conclusions would have been derived from looking at the holes. In this case, the hole concentration should be used in Eq. (10) instead of the electron concentration.

It is interesting to compare this time with the electron mobility lifetime in these semiconductors. This is done in Table 4 . The mobility in GaAs is thought to be limited by the phonon emission mechanism [22]. Although in this table it seems to be less frequent that the average number of collisions leading to the electron mobility, it has to be considered that the choice of $0.05 \mathrm{eV}$ for the electron temperature is rather arbitrary.

We have added to this table the matrix element calculated, somewhat arbitrarily, for a value $\left|\mathbf{k}-\mathbf{k}^{\prime}\right|^{2}=q^{2}=2 m_{e} k T_{a} / \hbar^{2}$. This matrix element is strongly dependent on $q$ being larger for small $q$. Anyway, even for a $q$ relatively large, the matrix element is rather large as compared to the multi-valley scattering case. The

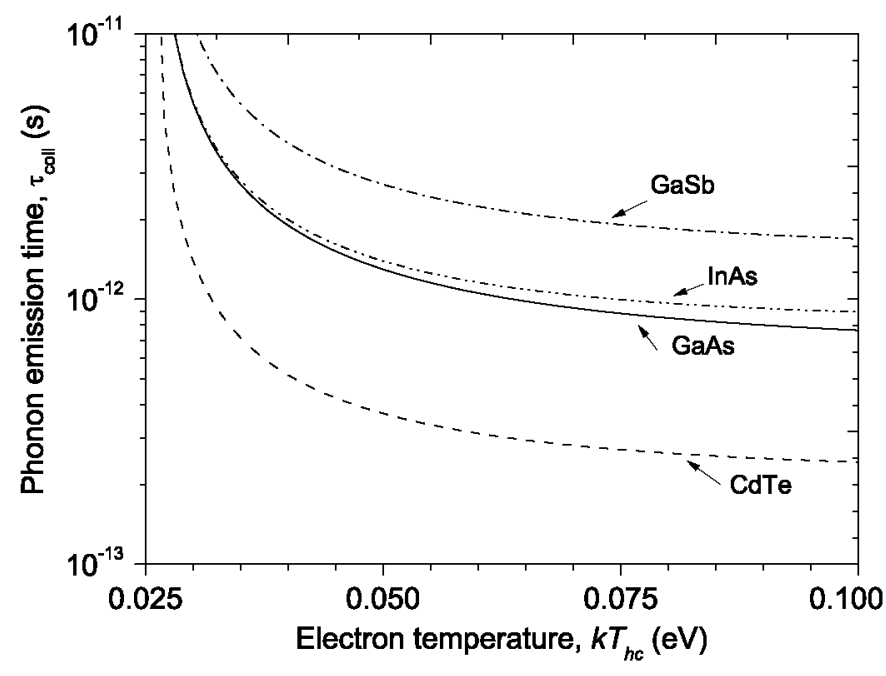

Fig. 5. Phonon emission time at phonon temperature $T_{a}=300 \mathrm{~K}$ calculated with parameters in Table 3 for the case of phonon scattering with conduction band electrons. Semiconductors are considered intrinsic.

reason why the lifetime is longer, and not shorter as it would be expected, should be surely attributed to the many modes for electrons available in the conduction band of the multi-valley case, more so that the effective mass is rather small, reducing again greatly the number of states occupied by electrons.

\section{Hot-carrier solar cell operation}

As presented in Fig. 6, a HC solar cell is made by sandwiching the low thermal interaction material between two metallic conductors and separated from them by dielectric layers with special mono-energetic contacts.

In Wurfel [5] these special contacts are called selective membranes (Fig. 6) that only allow electrons with energy centred around $\hat{E}_{e}$ (left contact) and $\hat{E}_{h}$ (right contact) to pass through. Impurity bands in dielectrics [25] or double barrier resonant structures based on quantum dots [26] may play the role given to these contacts. 


\begin{tabular}{|c|c|c|c|c|}
\hline & \multicolumn{4}{|l|}{ Material } \\
\hline & GaAs & InAs & GaSb & cdTe \\
\hline Electron mobilities at $300 \mathrm{~K}\left(\mathrm{~cm}^{2} \mathrm{~V}^{-1} \mathrm{~s}^{-1}\right)$ [20] & $8500[15]$ & $30000[24]$ & $5000[24]$ & $600[24]$ \\
\hline Mobility collision time $\tau=\mu m_{e} / e(s)$ & $3.24 \times 10^{-13}$ & $4.09 \times 10^{-13}$ & $1.17 \times 10^{-13}$ & $3.27 \times 10^{-14}$ \\
\hline Phonon emission time $\tau_{\text {coll }}$ at $k T_{e l}=0.05 \mathrm{e} . \mathrm{V} .(\mathrm{s})$ & $1.21 \times 10^{-12}$ & $1.39 \times 10^{-12}$ & $2.71 \times 10^{-12}$ & $3.71 \times 10^{-13}$ \\
\hline$\frac{2 \pi}{\hbar}\left|M_{\text {polar }}^{a b s}\left(\mathbf{k}, \mathbf{k}^{\prime}\right)\right|^{2}=\frac{e^{2} \hbar \omega}{4 \Omega \varepsilon_{0}}\left(\frac{1}{\varepsilon_{0 p t}}-\frac{1}{\varepsilon_{s t}}\right) \frac{\hbar^{2}}{2 m_{e}\left(k T_{0}\right)}\left(e . V . s^{-1}\right)$ & 0.0281 & 0.0127 & 0.00294 & 0.00666 \\
\hline
\end{tabular}

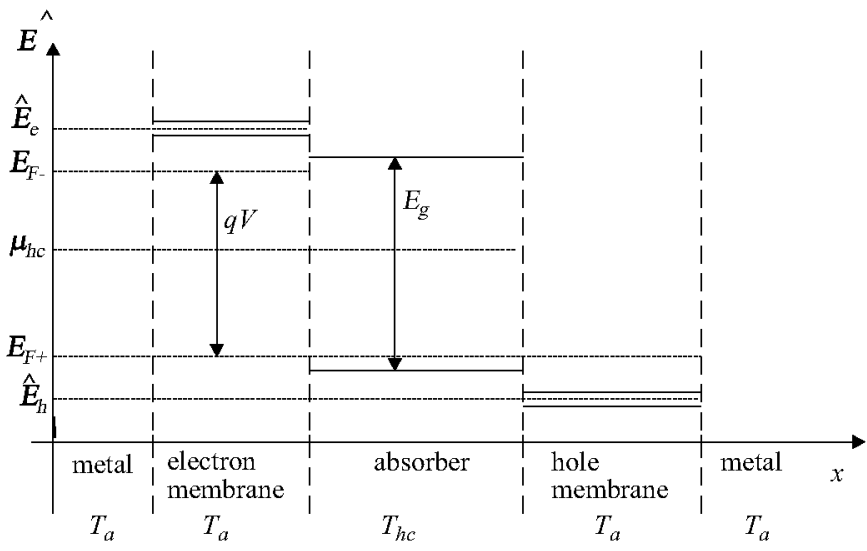

Fig. 6. Band structure of a hot-electron solar cell showing contacting scheme by means of selective membranes.

With reference to the nomenclature in Fig. 6 , the cell voltage is given by [5]

$q V=E_{F-}-E_{F+}=\left(\hat{E}_{e}-\hat{E}_{h}\right)\left(1-\frac{T_{a}}{T_{h c}}\right)$

The current extracted from the cell is determined by the rate at which electron hole pairs of energy $\hat{E}_{e}-\hat{E}_{h}$ can be withdrawn from the cell. Assuming that, additionally to this, a heat rate $\dot{Q}$ is gained by the electrons as heat delivered by the phonons (it is usually negative), the energy balance equation generalises the one in Ref. [5] and leads to

$I\left(\hat{E}_{e}-\hat{E}_{h}\right) / q=\dot{E}_{L}\left(T_{s}, 0, \varepsilon_{g}, \infty, H_{s}\right)-\dot{E}_{L}\left(T_{h c}, 0, \varepsilon_{g}, \infty, H_{r}\right)+\dot{Q}$

and the power extracted from the cell, can be finally computed as

$\dot{W}=I V=\left[\dot{E}_{L}\left(T_{s}, 0,0, \infty, H_{s}\right)\right.$

$$
\left.-\dot{E}_{L}\left(T_{h c}, 0,0, \infty, H_{r}\right)\right]\left(1-\frac{T_{a}}{T_{h c}}\right)+\dot{Q}\left(1-\frac{T_{a}}{T_{h c}}\right)
$$

In these two equations we use the energy flux corresponding to a beam of photons [3] given by

$\dot{E}_{L}\left(T, \mu, E_{m}, E_{M}, H\right)=\frac{2 H}{h^{3} c^{2}} \int_{E_{m}}^{E_{M}} \frac{E^{3} d E}{e^{\frac{E-\mu}{k T}}-1}$

being $T$ the photon temperature, $\mu$ their chemical potential, $E_{m}$ and $E_{M}$ the range of energies considered, $H$ the étendue or view factor $\left(H=\pi \sin ^{2} \gamma\right.$ for a bundle of rays incident in a cone of semi angle $\gamma$ ). $H_{s}$ is the etendue from the sun (solar view factor) whose angle depends on the concentration: it is about $0.26^{\circ}$ for no concentration and $\pi / 2$ for full concentration. $H_{r}$ is the étendue of the reradiated energy, usually with an angle $\pi / 2$.

Note that the power delivered, described by Eq. (19), is independent of the energy of the contacts. In other words, a large separation of the extracting energies lead to high voltage and low

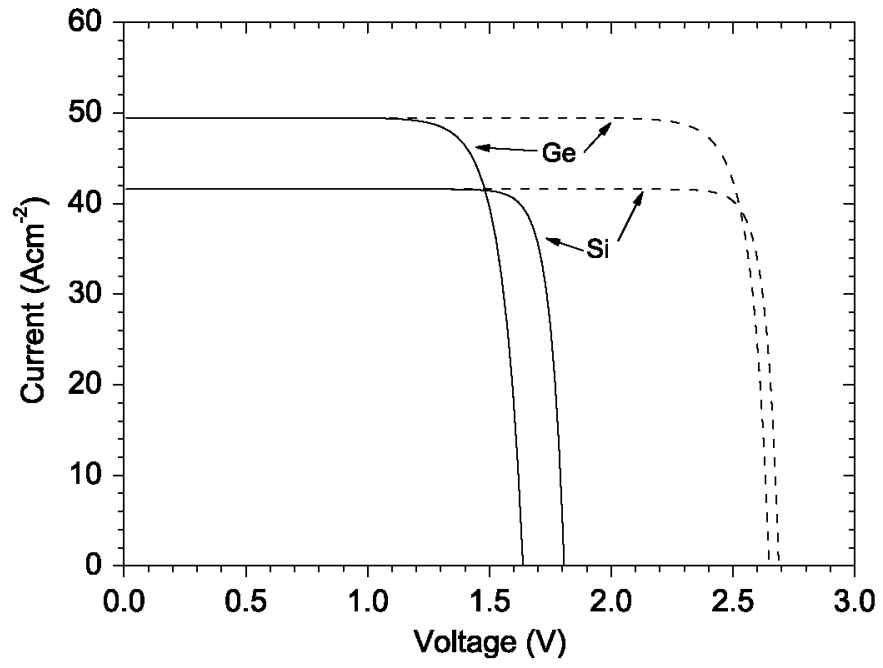

Fig. 7. Current-voltage characteristic of a hot carrier solar cell at 1000 suns (sun temperature $6000 \mathrm{~K}$, ambient $300 \mathrm{~K}$, solar view factor without concentration, $f_{S}=2.16 \times 10^{-5}$ ) with the narrow-energy contacts separated $3 \mathrm{eV}$. Solid curves correspond to the case with multi-valley thermal coupling. Dashed curves correspond to the ideal case (no electron-phonon coupling).

current and vice-versa. $T_{h c}$ is a parameter for this equation and also for the voltage Eq. (17). By elimination we obtain $W(V)$ and from it, the derived current-voltage curve. The cell efficiency is obtained from the maximum of $W(V)$ or $W\left(T_{h c}\right)$.

The power produced by this cell depends on $\dot{Q}$. In the ideal solar cell in Ref. [5] it is zero. However, it is a key element in assessing the performance of a less ideal HC solar cell.

In the preceding treatment, the existence of a band gap $E_{g}$ is not actually necessary for the $\mathrm{HC}$ solar cell, but, if it is made with a semiconductor with contacts at both sides of the band gap, an additional constraint appears associated to the conservation of the number of particles in each band [27]. The treatment becomes more complex and in some cases this limitation does not affect to the limiting efficiency involved in the present treatment. Since the purpose of this paper is to study the thermal coupling we do not consider it here.

This model can now be applied to the different materials we have analysed in the last section. We present in Fig. 7 the currentvoltage curves of a $\mathrm{HC}$ solar cell made of $\mathrm{Ge}$ and $\mathrm{Si}$ in thermal contact with the lattice trough the multi-valley scattering mechanism studied above and compare it with the cell without any thermal contact for the case of 1000 suns. For the rest, both cells are ideal (no contact losses, perfect light absorption, etc.). We see that the loss in efficiency corresponds to a drop from $84.1 \%$ to $48.1 \%$ for Ge and from $74.7 \%$ to $48.1 \%$ for $\mathrm{Si}$.

We present in Figs. 8 and 9 the efficiency at one-sun vs. the temperature for non-polar and polar semiconductors, 


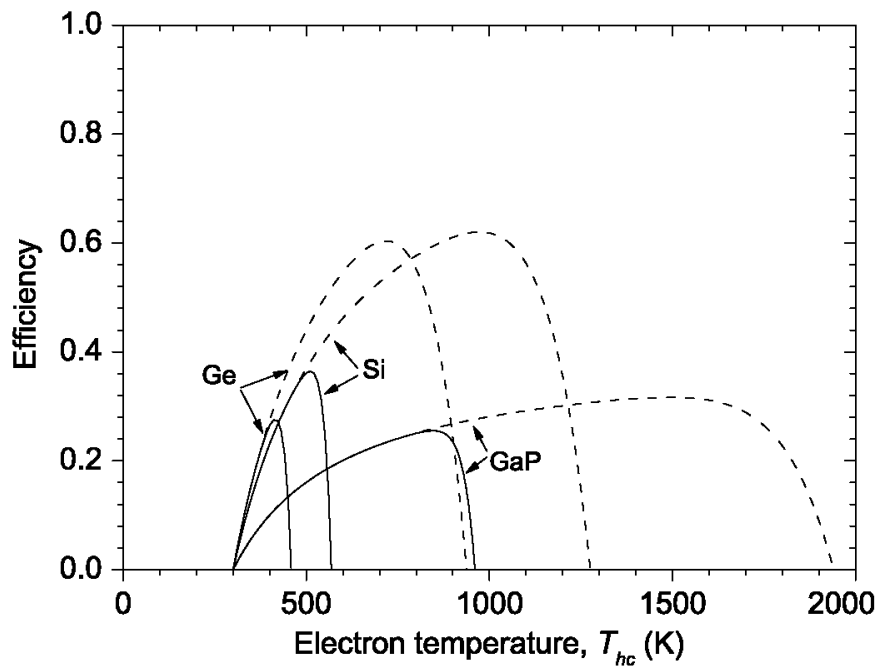

Fig. 8. Efficiency vs. electron temperature of a HC solar cell of indirect gap at one sun (sun temperature $6000 \mathrm{~K}$, ambient $300 \mathrm{~K}$, solar view factor $f_{S}=2.16 \times 10^{-5}$ ). Acceptor density is $5 \times 10^{18} \mathrm{~cm}^{-3}$. Solid lines take into account thermal losses. Dashed corresponds to the ideal case.

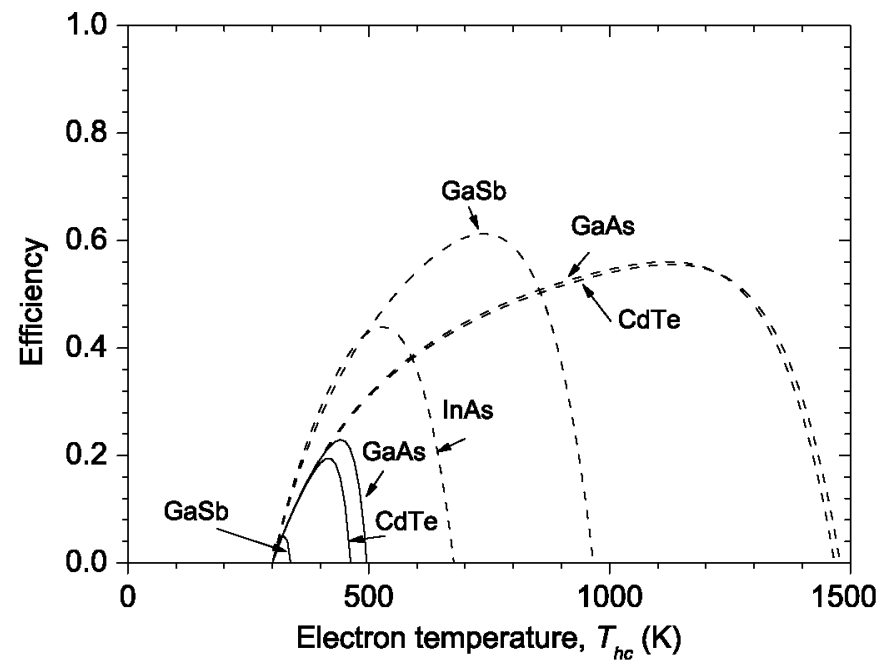

Fig. 9. Efficiency vs. electron temperature of HC solar cells in polar direct gap semiconductors at 1 sun (sun temperature $6000 \mathrm{~K}$, ambient $300 \mathrm{~K}$, solar view factor $f_{S}=2.16 \times 10^{-5}$ ). Semiconductors are intrinsic. Solid lines take into account thermal losses. Dashed corresponds to the ideal case. InAs is not visible when thermal coupling exists.

respectively, for all the materials we have studied in this work. In general it can be seen that the thermal contact is very small at low electron temperatures and only when the temperatures are high, the thermal coupling prevents the cells from reaching the efficiency maximum. This results, in general, in a reduction of the open circuit voltage, as seen in Fig. 7.

The best results are obtained for Si, with $36.5 \%$ vs. $60 \%$ for the fully ideal case. For the rest of the materials the performance is poorer, being worse for the polar semiconductors, mainly because there are more carriers as the Fermi level is near the gap centre. In particular InAs, with very small band gap, shows very low efficiency, being even its performance not visible in the plot, so that semimetals should be discarded for HC solar cell operation. Thermal-coupled performance of GaAs is better than that of CdTe, mostly due to less polar behaviour (the low- and high-frequency dielectric constants differ less) but still poorer that of the nonpolar semiconductors.

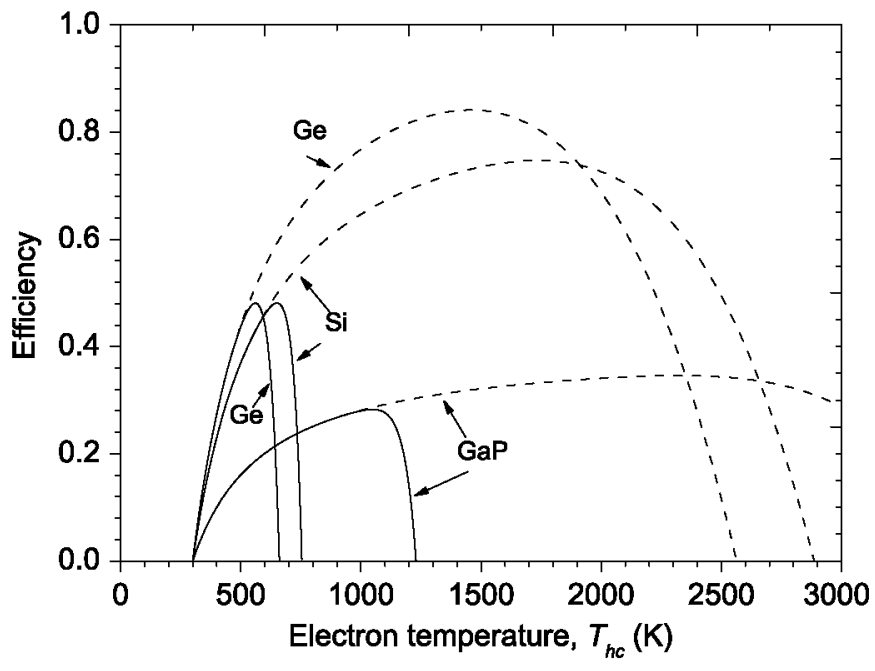

Fig. 10. Efficiency vs. electron temperature of $\mathrm{HC}$ solar cells in indirect gap semiconductors (sun temperature $6000 \mathrm{~K}$, ambient $300 \mathrm{~K}$ ) for a concentration of $1000 \times$ (solar view factor $f_{S}=2.16 \times 10^{-2}$ ). Acceptor density is $5 \times 10^{18} \mathrm{~cm}^{-3}$. Solid lines take into account thermal losses. Dashed lines correspond to the ideal case.

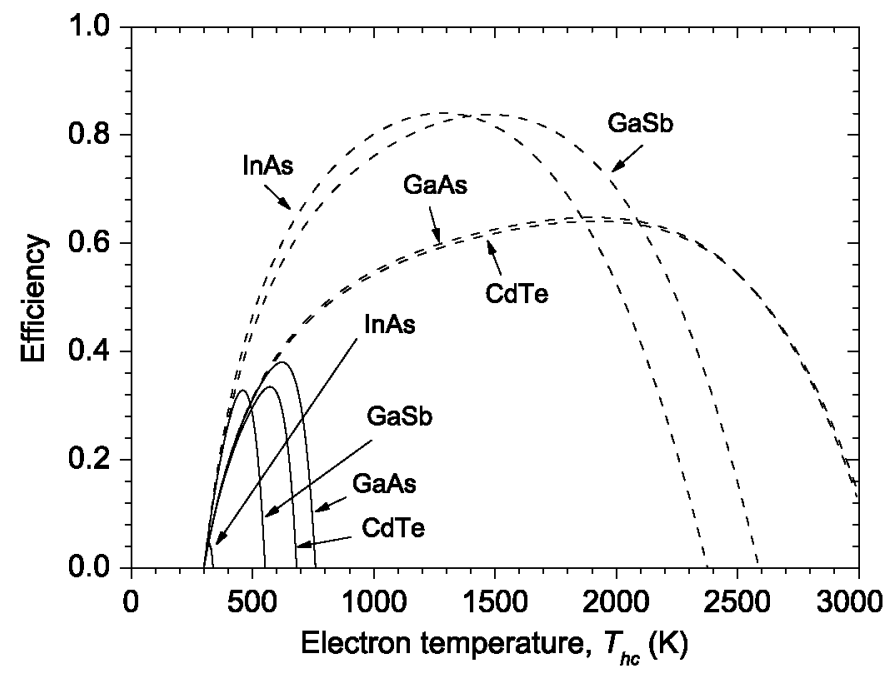

Fig. 11. Efficiency vs. electron temperature of $\mathrm{HC}$ solar cells in polar semiconductors (sun temperature $6000 \mathrm{~K}$, ambient $300 \mathrm{~K}$ ) for a concentration of $1000 \times$ (solar view factor $f_{S}=2.16 \times 10^{-2}$ ). Semiconductors are considered intrinsic. Solid lines take into account thermal losses. Dashed lines correspond to the ideal case. InAs is almost not visible with thermal coupling.

In Figs. 10 and 11 we present the efficiency under $1000 \times$ concentrated sunlight. Here $\mathrm{Ge}$ and $\mathrm{Si}$ cells present the same efficiency, 48.1\%, although in the ideal case, Ge presents higher efficiency due to its lower band gap, more adequate for concentration applications. Among the polar semiconductors, the best results are for the less polar ones, such a GaAs, but a too wide band gap is necessary to reduce the thermal coupling.

\section{Practical considerations}

The results and discussion above illustrate the potential of some well known semiconductors (for example silicon and germanium) to implement a $\mathrm{HC}$ solar cell. The limiting efficiencies calculated for a $\mathrm{HC}$ solar cell implemented with these materials are lower than the limiting efficiency of an abstract $\mathrm{HC}$ solar cell 
eventually implemented with a material not discovered yet. In any case, the theory described in this paper, which has established links between several parameters governing the mobility in a semiconductor and the rate heat is transferred from electrons to phonons, can be equally applied to $\mathrm{HC}$ solar cell material candidates that can be identified or proposed in the future once their mobility is empirically characterized.

Still, even if the material exhibits the appropriate potential, ways of implementing selective contacts of compatible technology with the material properties will have to be envisaged. As suggested by Conibeer et al. [25] and advanced above, these contacts could take the form of quantum dots. In the HC solar cell based on silicon discussed in this paper for example, these quantum dots could be implemented using a technology (basically the growth of $\mathrm{SiO}_{2}$ layers rich in $\mathrm{Si}$ followed by annealing) similar to the one that has been proposed for implementing allsilicon tandem solar cells [28].

\section{Conclusions}

We have developed a procedure to evaluate the thermal coupling of electrons to phonons in $\mathrm{HC}$ solar cells for the two processes that are deemed inelastic and that affect importantly the carrier mobility, namely multi-valley scattering in indirect band gap semiconductors and optical phonon coupling in polar semiconductors. The procedure has been applied to several indirect gap and polar direct gap semiconductors. In all cases it has been found that the coupling is proportional to the density of carriers. It increases with the voltage and therefore the open circuit voltage is reduced with respect to the ideal cases without thermal coupling.

Since multi-valley scattering is associated to electrons in the conduction band, it is possible to p-dope heavily the semiconductors so that the electron density in the conduction band is minimized and therefore the effect of the thermal coupling is also minimized. In consequence, heavily doped p-type Ge, given the long phonon emission time we have calculated for it, that is associated with the high measured mobility, and $\mathrm{Si}$, due to its larger band gap that reduces the density of electrons when the acceptor doping is high enough, result the best candidates with efficiency limit of $48.1 \%$ at 1000 suns in both cases after taking into account the thermal losses. At 1 sun, $\mathrm{Si}$ is the best material with efficiency limit 36.5\%. The rest of the materials perform worse. GaP, which is polar, but with indirect gap is included in this group. It is already discarded without studying it as polar semiconductor what would require a special treatment due to the indirect nature of its gap.

Indeed, the relatively good performance of $\mathrm{Si}$ and, in concentration, Ge cells strongly rely on the elastic nature of the scattering for the holes, of high density in this option. Their conductivity is mostly limited by acoustic phonons and ionized and non-ionized impurity scattering, and all these processes are deemed to be elastic. If so, no thermal coupling should be produced. But the subject should be re-examined carefully.

For the polar semiconductors, with direct band gaps, the coupling with longitudinal optical phonons is the main limitation to mobility. We have found that the results are poorer. The main reason is that, in this case, both electrons and holes, can emit phonons so that the recommendation is to work with nearly intrinsic semiconductors. This reduces electrons and hole densities less efficiently (Fermi level has to be located near the mid of the band gap) and the coupling with the phonons remains high. Thus, polar semiconductors are not attractive for this application.

It has been suggested $[9,10]$ that, since thermal coupling is produced with optical phonons, some materials like InN with large phononic band gap $[7,8]$ might have a poor thermal coupling between optical and acoustic phonons. This behaviour has been also related to the phonon bottleneck in low-dimensional structures. The concept is attractive and in addition most probably $\mathrm{Si}, \mathrm{Ge}$ and other classic materials have a too strong thermal coupling so that novel solutions have to be sought. In any case the decoupling of the optical and acoustical phonon temperatures relies in the low thermal conductivity attributed to the optical phonons. This property has to be examined because the dispersion curve of the optical phonons is horizontal only in a first order study and optical phonons have small but not zero group velocity so that the thermal conductivity may not be negligible.

\section{Acknowledgments}

This work has been supported by the Global Climate and Energy Project (GCEP).

\section{References}

[1] W. Shockley, H.J. Queisser, Detailed balance limit of efficiency of p-n junction solar cells, Journal of Applied Physics 32 (1961) 510-519.

[2] G.L. Araujo, A. Marti, Absolute limiting efficiencies for photovoltaic energy conversion, Solar Energy Materials and Solar Cells 33 (1994) 213-240.

[3] A. Luque, A. Marti, Theoretical limits of photovoltaic energy conversion, in: A. Luque, S. Hegedus (Eds.), Handbook of Photovoltaic Science and Engineering, John Wiley \& Sons, Chichester, 2003, pp. 113-151.

[4] R.T. Ross, A.J. Nozik, Efficiency of hot-carrier solar energy converters, Journal of Applied Physics 53 (1982) 3813-3818.

[5] P. Wurfel, Solar energy conversion with hot electrons from impact ionisation, Solar Energy Materials and Solar Cells 46 (1997) 43-52.

[6] J. Urayama, T.B. Norris, J. Singh, P. Bhattacharya, Observation of phonon bottleneck in quantum dot electronic relaxation, Physical Review Letters 86 (2001) 4930.

[7] VY Davydov, VV Emtsev, IN Goncharuk, AN Smirnov, V.D. Petrikov, VV. Mamutin, V.A. Vekshin, S.V. Ivanov, M.B. Smirnov, T. Inushima, Experimental and theoretical studies of phonons in hexagonal InN, Applied Physics Letters 75 (1999) 3297-3299.

[8] C. Bungaro, K. Rapcewicz, J. Bernholc, Ab initio phonon dispersions of wurtzite AlN, GaN, and InN, Physical Review B 61 (2000) 6720-6725.

[9] G.J. Conibeer, J.F. Guillemoles, M.A. Green, Phononic band gap engineering for hot carrier solar cell absorbers, in: WIP (Ed.), Proceedings of the 20th European Photovoltaic Solar Energy Conference, Barcelona, Spain, 2005, pp. 35-38.

[10] G.J. Conibeer, J.F. Guillemoles, D. König, M.A. Green, Phononic engineering for hot carrier solar cells including interface modelling, in: Proceedings of the 21 st European Photovoltaic Solar Energy Conference, WIP, Dresden, Germany, 2006, pp. 90-93.

[11] P.G. Klemens, Anharmonic Decay of Optical Phonons, Physical Review 148 (1966) 845-848.

[12] G.P. Srivastava., The Physics of Phonons, Taylor and Francis, New York, 1990.

[13] J.B. Gunn, Microwave oscillations of current in III-V semiconductors, Solid State Communications 1 (1963) 88-91.

[14] W.T. Read, A proposed high-frequency, negative-resistance diode, Bell System Technical Journal 37 (1958) 401-446.

[15] K.W. Boer, Survey of Semiconductor Physics, Van Nostrand Reinhold, New York, 1990

[16] E. Conwell, V.F. Weisskopf, Theory of Impurity Scattering in Semiconductors, Physical Review 77 (1950) 388-390.

[17] J. Bardeen, W. Shockley, Deformation potentials and mobilities in non-polar crystals, Physical Review 80 (1950) 72-80.

[18] W. Shockley, J. Bardeen, Energy bands and mobilities in monatomic semiconductors, Physical Review 77 (1950) 407-408.

[19] C. Herring, Transport properties of a many-valley semiconductor, Bell System Technical Journal 34 (1955) 237-290.

[20] D.L. Rode, Electron mobility in Ge, Si, and Gap, Physica Status Solidi B-Basic Research 53 (1972) 245-254.

[21] H. Ehrenreich, Electron scattering in Insb, Journal of Physics and Chemistry of Solids 2 (1957) 131-149.

[22] K. Fletcher, P.N. Butcher, Exact solution of linearized boltzmann-equation with applications to hall-mobility and hall factor of GaAs, Journal of Physics Part C Solid State Physics 5 (1972) 212-224.

[23] E. Yablonovitch, G.D. Cody, Intensity enhancement in textured optical sheets for solar cells, IEEE Transactions on Electron Devices 29 (1982) 300-305

[24] R.A. Smith, Semiconductors, Cambridge University Press, London, 1968.

[25] G. Conibeer, C.W. Jiang, M. Green, N. Harder, A. Straub, Selective energy contacts for potential application to hot carrier PV cells, in: K. Kurokawa, L.L Kazmerski, B. McNelis, M. Yamaguchi, C. Wronski, W.C. Sinke (Eds.), 
Proceedings of Third World Conference on Photovaltaic Energy Conversion, vols a-C, 2003, pp. 2730-2733.

[26] G.J. Conibeer, C.W. Jiang, D. König, S. Shrestha, T. Walsh, M.A. Green, Selective energy contacts for hot carrier solar cells, Thin Solid Films 516 (2008) $6968-6973$.
[27] P. Wurfel, A.S. Brown, T.E. Humphrey, M.A. Green, Particle conservation in the hot-carrier solar cell, Progress in Photovoltaics 13 (2005) 277-285.

[28] I. Perez-Wurfl, X. Hao, A. Gentle, D.-H. Kim, G. Conibeer, M.A. Green, Si nanocrystal p-i-n diodes fabricated on quartz substrates for third generation solar cell applications, Applied Physics Letters 95 (2009) 153506 (3pp.). 